\title{
Multivariate Downside Risk: Normal versus Variance Gamma
}

\author{
Martin Wallmeier, Martin Diethelm*
}

February 2011

\begin{abstract}
Although several types of options on multiple assets are popular in today's financial markets, valuing multi-asset options is still a challenge in finance. The standard framework of multivariate normality is often inappropriate, since it ignores fat tails and other stylized facts of asset returns. The Variance Gamma (VG) model appears to be a promising alternative. In the univariate case, it has become a standard tool in finance. The traditional way to extend the model to the multivariate case is to subordinate a Brownian motion through a univariate subordinator. In recent years, generalizations with multivariate subordinators have been proposed. Our objective is to study two versions of the multivariate VG model in a large-scale application with multi-asset options traded in an active market. Our database consists of 468 multivariate barrier reverse convertibles at the Swiss market for structured products. The Swiss market ranks among the largest in the world and is characterized by an exceptional popularity of multiple asset options. We find that there is a trade-off between the two VG models considered: one performs better in capturing the smile, the other is more often able to capture the correlation structure. In all, based on our calibration, only 316 out of 468 products can be evaluated with at least one of the two VG models. We conclude that there is a need for more flexible extensions.
\end{abstract}

JEL classification: G13; G15; G14

Keywords: Lévy Process, Variance Gamma Process, Multi-Asset Options, Barrier Options, Reverse Convertibles, Structured Financial Products.

${ }^{*}$ Prof. Dr. Martin Wallmeier (corresponding author), Martin Diethelm, Department of Finance and Accounting, University of Fribourg / Switzerland, Bd. de Pérolles 90, CH-1700 Fribourg, Tel. +41 26300 8294, Fax +41 26300 9659, martin.wallmeier@unifr.ch 


\section{Introduction}

Valuing options on multiple assets is still a challenge in finance (see Leoni and Schoutens (2008)). Solutions are readily available within the standard framework of multivariate normality, but this ignores heavy tails and other characteristics of empirical return distributions. The normality assumption is too restrictive, especially in cases when the payoff strongly depends on the downside risk of the underlying assets. For instance, one of the most successful types of instruments at the Swiss market for structured financial products is a barrier reverse convertible on multiple assets. It consists of buying a straight bond and at the same time selling a multi-asset put option. Although the product has passed the test of acceptance by the investing public long ago, it is still not clear which valuation model to use and how to consider heavy tails. Most investors in multiple barrier reverse convertibles incurred unexpected losses during the recent financial crisis, and there is some evidence that they had underestimated the risk involved in the instruments (see Lindauer and Seiz (2008), Wallmeier and Diethelm (2009)). ${ }^{1}$ The weaknesses of the standard normal valuation approach have become more obvious than ever during the last two years. ${ }^{2}$

In the univariate case, generalized hyperbolic distributions have often been proposed as an alternative. ${ }^{3}$ They can capture three important characteristics of stock returns, which are nonnormality, time-varying volatility and the leverage effect. Since a distribution of this class is infinitely divisible, a corresponding Lévy process always exists (see, e.g., Bibby and Sørensen (2003)). Lévy processes have generally proven to be a better basis for option valuation than the standard assumption of geometric Brownian motion (GBM). ${ }^{4}$

A special case of generalized hyperbolic processes is the Variance Gamma (VG) process which was introduced by Madan et al. (1998), based on earlier work of Madan and Seneta (1990) and Madan and Milne (1991). In the VG model, Brownian motion is evaluated at a random time change given by a Gamma process. The VG process includes two additional parameters compared to Brownian motion, which together provide control over the skewness and kurtosis of the return distribution. Apart from the Normal Inverse Gaussian (NIG) process (see BarndorffNielsen (1997), Barndorff-Nielsen (1998)), the VG process is the only generalized hyperbolic process which is closed under convolution so that the stock price at any point in time is VG distributed (see, e.g., Bibby and Sørensen (2003)). Therefore, it is a natural candidate for

1 Hens and Rieger (2008) show that some of the most successful structured products cannot be optimal for rational investors; see similarly Henderson and Pearson (2009).

2 See Chen et al. (2002) and Boyle et al. (1989) for numerical valuation of multi-asset options under the normal approach. See Geman (2002) for a review of fundamental properties of Lévy processes.

3 See, e.g. Eberlein and Keller (1995), Eberlein et al. (1998), Rydberg (1999), Raible (2000), Barndorff-Nielsen and Shephard (2001), Schoutens (2003), Cont and Tankov (2004), Applebaum (2004), Fiorani (2004).

4 See, e.g., Eberlein et al. (1998); Madan et al. (1998); Lam et al. (2002); Carr and Wu (2004); Rathgeber (2007). 
modelling financial returns. Since robust estimation methods are available, it has become a standard model in the financial industry (see Carr et al. (2007), Carr and Madan (1999)). ${ }^{5}$

The situation is different in the multivariate case. The traditional way to construct a multivariate Lévy process is to subordinate a Brownian motion through a univariate subordinator (see, e.g., Monroe (1978)). Following this approach, Luciano and Schoutens (2006) propose a common Gamma subordinator and an uncorrelated multidimensional Brownian motion. Cont and Tankov (2004) and Leoni and Schoutens (2008) extend this model to correlated Brownian motions, which allows for uncorrelated asset returns. One drawback of these models is that the time change is assumed to be the same for all assets, which is contrary to economic intuition. Another problem is that the achievable range of dependence is limited. For example, asset returns cannot be independent in this approach. Recent research has focused on alleviating these problems by using multivariate subordinators (see Semeraro (2008), Ballotta (2008), Marfè (2009), Luciano and Semeraro (2010a, 2010b). These models offer more flexibility with respect to the VG marginal distribution and improve modelling of the dependence structure, at least when they do not rely on the assumption of independent Brownian motions.

To our knowledge, a comprehensive study on smile-consistent calibration of multivariate Lévy processes for a sample of multi-asset financial instruments traded in an active market has not yet been published. Thus, it is difficult to assess the suitability of these models in standard applications. This is where we aim to contribute to the literature. Our database consists of 468 multivariate barrier reverse convertibles. They are traded at the Swiss market for structured products which ranks among the largest in the world and is characterized by an exceptional popularity of multiple asset options. For the multivariate VG model to be applicable in large-scale applications, implementation is an important issue. This is why we focus on two parsimonious models: (1) the model of Leoni and Schoutens (2008) with a univariate subordinator and correlated Brownian motions, and (2) the $\alpha$-VG model of Semeraro (2008) with a multivariate subordinator and uncorrelated Brownian motions. The idea is to check if these rather simple versions are sufficient to consistently evaluate market instruments. In case of a negative answer, generalizations as proposed by Marfè (2009) and Luciano and Semeraro (2010a, 2010b) could be applied.

Apart from the empirical analysis of the multivariate VG model, our study aims to contribute to the literature on the pricing of structured financial products. The existing studies typically derive fair values from the multivariate normal model (see, e.g., Hernandez et al. (2007), Szymanowska et al. (2009)). However, ignoring fat tails and other characteristics might seriously bias the results on the degree of overpricing.

The remainder of the paper is structured as follows: Section 2 presents the two versions of the multivariate VG model and illustrates the implications for the smile in option prices. In Section

5 The CGMY process proposed by Carr et al. (2002) generalizes the VG process by adding an additional parameter. 
3 , we perform a simulation analysis to reveal the sources of mismatch between the Gaussian and the VG model in valuing multiple barrier reverse convertibles. Section 4 presents the empirical study and Section 5 concludes.

\section{Multivariate Variance Gamma Model}

\subsection{Univariate subordinator}

The traditional way to construct a multivariate Lévy process $\mathbf{X}=\{\mathbf{X}(t), t \geq 0\}$ is to subordinate a multivariate Brownian motion with a univariate subordinator. In the case of the Variance Gamma (VG) process, the subordinator is Gamma distributed. Let $\mathbf{X}(\mathbf{0})=0$, and let the increment, $\mathbf{X}(\mathbf{s}+\mathbf{t})-\mathbf{X}(\mathbf{s})$, over any time interval of length $t$ have the same distribution as $\mathbf{X}(\mathbf{t})$. Then, the multivariate VG process can be written as:

$$
\mathbf{X}(t)=t \mu+\theta V(t)+\mathbf{W}(V(t))
$$

where $\mu, \theta$ are $n$-dimensional parameter vectors, $V(t) \sim G a\left(t \frac{1}{\nu}, \frac{1}{\nu}\right)$ is a random variable following a Gamma distribution with variance rate $\nu$, and $\mathbf{W}(V(t))$ is a Gamma time-changed Brownian motion, i.e. $d \mathbf{W}(V(t))=\varepsilon \sqrt{d V(t)}$, where $\varepsilon$ is a vector of random normally distributed variables with covariance matrix $\boldsymbol{\Sigma}$. We denote the variances on the main diagonal of $\boldsymbol{\Sigma}$ by $\omega_{k}^{2}(k=$ $1, \ldots, n)$. The variable $\mathbf{X}(t)$, which is interpreted as log stock return or log stock price, is Variance Gamma distributed:

$$
\mathbf{X}(\mathbf{t}) \sim V G(t \mu, t \theta, \nu / t, t \boldsymbol{\Sigma})
$$

with expected value $E(\mathbf{X}(t))=t \mu+t \theta$ and covariance matrix $\operatorname{Cov}(\mathbf{X}(t))=t \boldsymbol{\Sigma}+t \nu \theta \theta^{\prime}$.

This VG model, in the following denoted by UNI-VG, is an extension of the original model of Madan and Seneta (1990) and is used by Leoni and Schoutens (2008). There are two important limitations of this approach. First, the assumption that all asset prices jump at the same time is difficult to justify on economic grounds. The information arrival reflected in trading activity may have a common component, but will also be influenced by stock-specific events. ${ }^{6}$ The second disadvantage is the limited range of correlations achievable within the model. In particular, low correlations are not compatible with the model if the variance rate $\nu$ is high and all stocks exhibit the typical leverage effect with negative $\theta$ coefficients.

In the theoretical literature, several models have been proposed to overcome these problems. One immediate alternative to the Leoni and Schoutens (2008) approach is the $\alpha$-VG model of Semeraro (2008) (see also Luciano and Semeraro (2010b) and Luciano and Semeraro (2010a)),

\footnotetext{
6 Time changes are understood as a way to model the information flow which is reflected in the number of trading transactions, see Ané and Geman (1996), Ané and Geman (2000).
} 
which assumes independent Brownian motions but allows for different time changes for the assets. In this way, the model extends the original Madan and Seneta (1990) approach in a different direction than UNI-VG. The $\alpha$-VG model is parsimonious, easy to calibrate and economically well founded since it allows for stock-specific information arrival. ${ }^{7}$ For these reasons, we include the $\alpha$-VG model in our empirical analysis. The model is introduced in the next section.

\subsection{Multivariate subordinator: $\alpha$-VG model}

Let $A_{i}=\left\{A_{i}(t), t \geq 0\right\}, i=1, \ldots, n$ and $Z=\{Z(t), t \geq 0\}$ be independent Gamma processes with distributions $A_{i}(t) \sim G a\left(t\left(\frac{1}{\alpha_{i}}-a\right), \frac{1}{\alpha_{i}}\right)$ and $Z(t) \sim G a(t a, 1)$ where $0<\alpha_{i}<\frac{1}{a}$. Then, the processes $G_{i}=\left\{G_{i}(t), t \geq 0\right\}$ defined by:

$$
G_{i}(t)=A_{i}(t)+\alpha_{i} Z(t)
$$

can be used as multivariate subordinator which is again Gamma distributed: $G_{i}(t) \sim G a\left(t \frac{1}{\alpha_{i}}, \frac{1}{\alpha_{i}}\right)$. The economic intuition is that the time change can be decomposed into a common component and a stock-specific component.

Based on this subordinator, the $n$-dimensional Lévy process $\mathbf{Y}=\{\mathbf{Y}(t), t \geq 0\}$ of the $\alpha$-VG model is defined as:

$$
\mathbf{Y}(t)=\left(\begin{array}{c}
Y_{1}(t) \\
\ldots \\
Y_{n}(t)
\end{array}\right)=\left(\begin{array}{c}
\mu_{1}+\theta_{1} G_{1}(t)+\omega_{1} W_{1}\left(G_{1}(t)\right) \\
\ldots \\
\mu_{n}+\theta_{n} G_{n}(t)+\omega_{n} W_{n}\left(G_{n}(t)\right)
\end{array}\right)
$$

where $W_{i}=\left\{W_{i}(t), t \geq 0\right\}, i=1, \ldots, n$ are uncorrelated standard Brownian motions. The expected values, variances and covariances are $E\left(Y_{i}(t)\right)=t \mu_{i}+t \theta_{i}, \operatorname{Var}\left(Y_{i}(t)\right)=\omega_{i}^{2}+\alpha_{i} \theta_{i}^{2}$, and $\operatorname{Cov}\left(Y_{i}(t), Y_{j}(t)\right)=a \theta_{i} \theta_{j} \alpha_{i} \alpha_{j}, i \neq j$.

Within the limits of $0<a<\min \left(\frac{1}{\alpha_{1}}, \ldots, \frac{1}{\alpha_{n}}\right)$, the paramater $a$ can be used to adjust the level of correlations to empirical estimates. However, the same parameter $a$ applies to all pairs of stocks, which means that the structure of the correlation matrix is fully determined by parameters $\theta$ and $\alpha$. Low (positive) correlations can easily be achieved by setting $a$ to a value near zero, which is an important advantage compared to the alternative UNI-VG model (see Section 2.1). However, the upside limit will be rather restrictive if one of the $\alpha_{i}$-coefficients is high. The relevance of this upper limit of $a$ can only be evaluated empirically. In contrast to the UNI-VG model, it is not possible to increase the range of dependence by using correlated Brownian motions, because the resulting process would no longer be part of the Lévy class (see Luciano and Semeraro (2010b)).

\footnotetext{
$\overline{7}$ For an application of the $\alpha$-VG model in the filed of credit risk see Fiorani et al. (2010).
} 


\subsection{Implications for Option Smile (one underlying asset)}

Following Leoni and Schoutens (2008), we use the mean-adjusting equivalent martingale measure to evaluate options under VG stock price processes. Figure 1 illustrates smile patterns for different parameter sets. They are based on a variance rate of the Gamma process of $\nu=0.1$ and 0.25 , respectively (notation from UNI-VG model with one underlying asset). Parameter $\theta$ is chosen from $\theta \in\{-0.2,-0.5\}$, parameter $\omega$ from $\omega \in\{0.1,0.2\}$. In order to illustrate the smile surface, each graph in Figure 1 shows the smile pattern for different times to maturity $T$, where $T \in\{0.125,0.25,0.5,1.0\}$ (in years).

It is important to note that there is no one-to-one correspondence between parameters $\omega, \theta, \nu$ and volatility, skewness and kurtosis. The following relationships hold:

$$
\begin{aligned}
E\left[\left(X_{t}-E\left(X_{t}\right)\right)^{2}\right] & =\left(\omega^{2}+\nu \theta^{2}\right) t \\
E\left[\left(X_{t}-E\left(X_{t}\right)\right)^{3}\right] & =\left(2 \theta^{3} \nu^{2}+3 \omega^{2} \theta \nu\right) t \\
E\left[\left(X_{t}-E\left(X_{t}\right)\right)^{4}\right] & =\left(3 \omega^{4} \nu+12 \omega^{2} \theta^{2} \nu^{2}+6 \theta^{4} \nu^{3}\right) t \\
& +\left(3 \omega^{4}+6 \omega^{2} \theta^{2} \nu+3 \theta^{4} \nu^{2}\right) t^{2}
\end{aligned}
$$

This means that the influences of the single parameters on the smile in option prices will be interrelated. Nevertheless, we can highlight some general observations:

It is apparent that the smile or skew pattern is more pronounced for short-dated options and flattens out for longer times to maturity. For $T=1$, the pattern is almost linear and very similar across the different parameter sets. The other parameters also have the expected influence. In particular, $\theta$ strongly influences the asymmetry of the pattern between low and high strike prices. Given this asymmetry, the skew becomes more pronounced with increasing $\nu$. An increase in $\omega$ mainly results in an upward shift of the smile pattern.

Overall, the graphs show that the typical skew and smile patterns prevailing in single stock options can be reproduced with appropriate choices of the VG parameters. This is not the case for the Black-Scholes model which implies a flat line of implied volatilities across moneyness. Therefore, compared to Black-Scholes, the VG model will almost surely be more in line with observed option prices. However, there is an important difference between UNI-VG and alphaVG. In the UNI-VG model, the variance rate $\nu$ is a common parameter, which means that $\nu$ is forced to be equal for all underlying assets. This is a severe restriction in the empirical implementation, the more so as there is no economic reason why the parameter should be the same for each stock. To illustrate the problem, let us assume that the marginal smile of the first underlying stock corresponds to the upper left graph of Figure 1, while the smile pattern of the second underlying stock corresponds to the upper right graph. Thus, to accurately capture the smile patterns, we would have to choose $\nu_{1}=0.1$ for the first stock and $\nu_{2}=0.25$ for the second. But this is excluded in the UNI-VG model. Forcing $\nu$ to be the same for all stocks will 
result in inaccurate smile patterns. The alpha-VG model does not have this restriction as it allows a different $\nu$ parameter for each stock. As a consequence, almost by definition, the fit of the alpha-VG model will be better at the marginal level. In the empirical part (Section 4), we show that this is an important advantage of alpha-VG compared to UNI-VG.

\section{Valuation of Multiple Barrier Reverse Convertibles (MBRC)}

\subsection{Payoff Profile}

The Swiss market for structured financial products ranks among the largest in the world. Its market value represents about $5 \%$ of financial wealth managed in Switzerland. ${ }^{8}$ Structured products consist of two or more different components, one of which must be a derivative (see Stoimenov and Wilkens (2005)). They are issued by banks and may be addressed to private or institutional investors. The products can be traded on an organized exchange or sold directly by their issuing bank, who will quote bid and ask prices. A unique characteristic of the Swiss market is that structured products with "exotic" options are extremely prominent. In particular, barrier reverse convertibles represent more than one third of the market (in terms of the total number of products). The majority of these contain multi-asset options, and are called Multiple Barrier Reverse Convertibles (MBRC). ${ }^{9}$ In other countries, an active market only exists for simpler types of reverse convertibles. Thus, the Swiss market offers an exceptional opportunity to study the pricing of multi-asset instruments.

The buyer of an MBRC firstly receives a fixed coupon payment on the nominal value of the product. Secondly, at maturity, the issuing bank will either repay the nominal value or deliver a certain number of stocks. It has to repay the nominal value, if during the product's lifetime none of the prices of the underlying assets has hit a contractually specified downside barrier or at maturity all asset prices are above their initial values. If none of these conditions is fulfilled, i.e. if one of the barriers has been broken and one of the final asset prices is below initial value, the issuing bank will deliver the underlying asset with the worst performance (relative return). The number of stocks to be delivered is fixed in the contract. It is set such that their total market value is equal to the nominal contract value in the case of zero stock return. This design means that the investor's nominal capital is protected as long as none of the barriers is hit (see broken line in Figure 2), even if one of the stock prices is below strike at maturity. However, as soon as one barrier is triggered, the MBRC turns into a regular reverse convertible on multiple underlying assets (see the unbroken and dotted lines in Figure 2).

\footnotetext{
8 See the market statistics of Scoach by SIX Group and Deutsche Börse: www.scoach.ch.

9 These have been heavily promoted by some issuers. In 2007, more than 700 full-page advertisements appeared in the most respected Swiss newspaper "Neue Zürcher Zeitung" and the business paper "Finanz und Wirtschaft".
} 

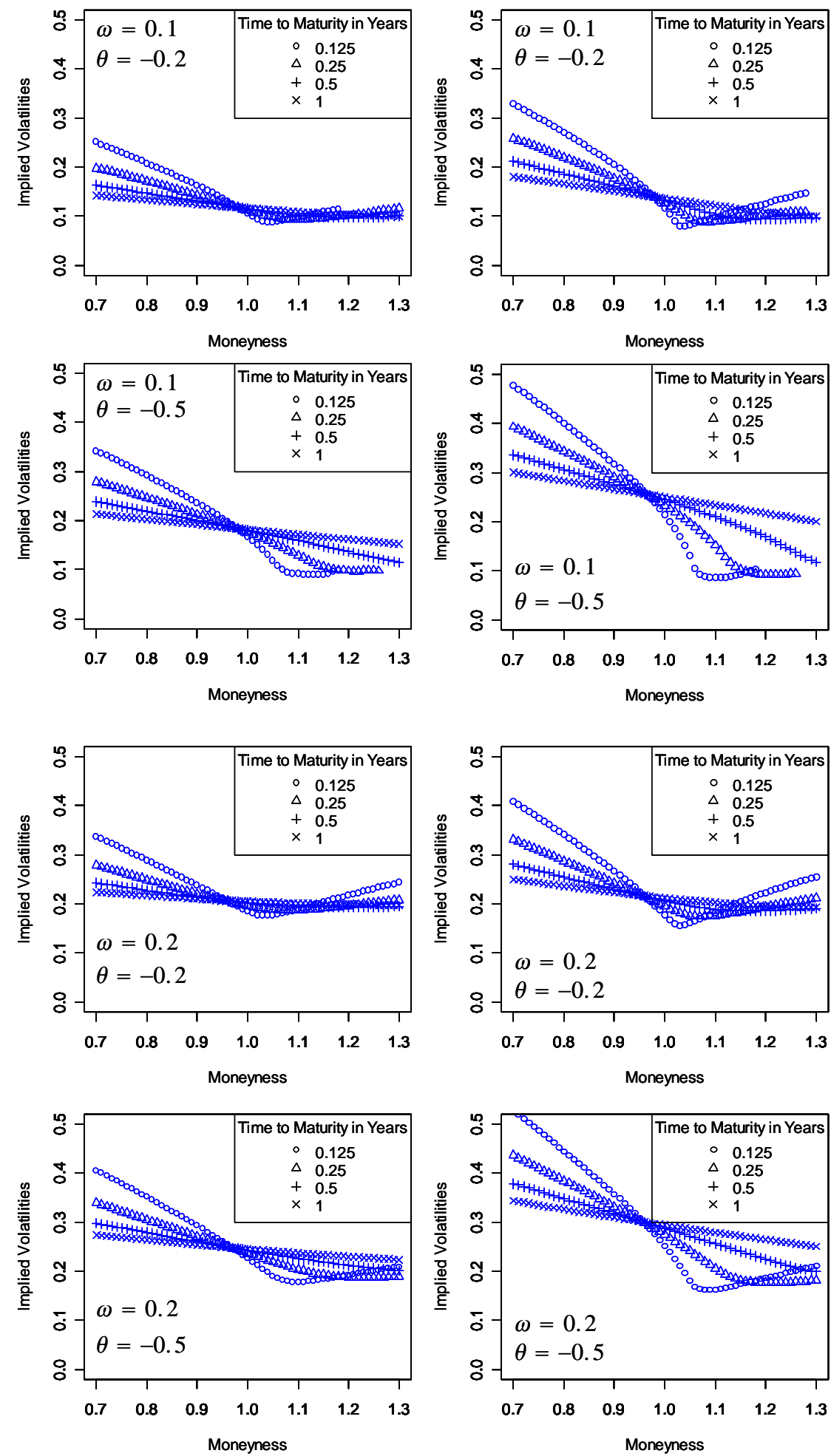

Figure 1: Smile patterns for various Variance Gamma parameters. Graphs on the left are based on $\nu=0.1$; graphs on the right are based on $\nu=0.25$. 


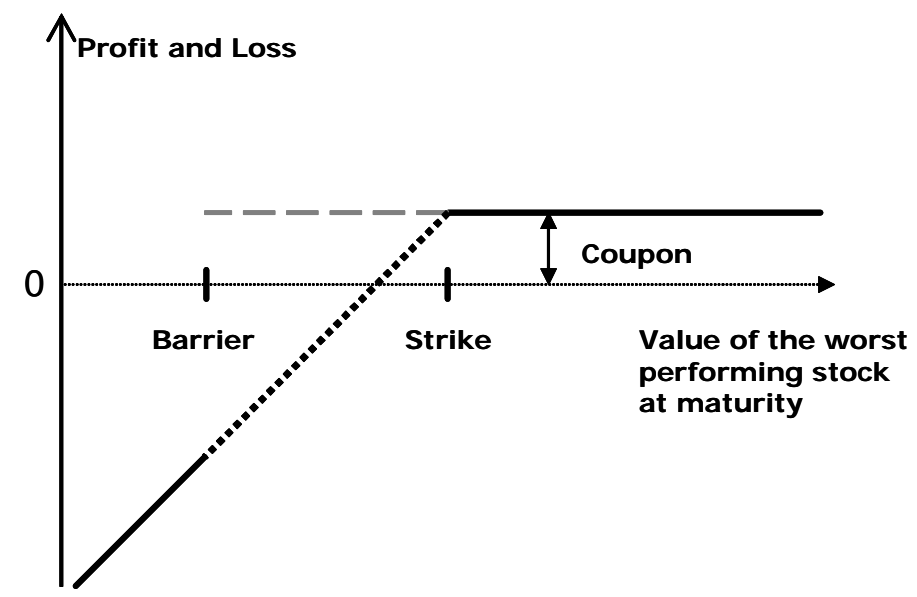

Figure 2: Profit and loss diagram of multi barrier reverse convertibles; dotted line: relevant if barrier has been triggered; broken line: relevant if barrier has not been triggered

To summarize, an MBRC is a combination of a straight bond with a written put option. The put is 'exotic' because it is based on more than one underlying asset and includes a downside barrier. The option premium corresponds to the coupon surplus over the risk-adjusted bond yield:

Option premium paid $=$ Present value of coupon payments above bond yields.

In our empirical study, we focus only on the option component and compare the option premium paid in the market to the fair option value according to the VG model.

\subsection{VG Parameters and MBRC Values in the UNI-VG model}

Before analyzing the empirical data, it is of interest to gain insight into the general relationship between VG parameters and fair values of MBRC. We perform this analysis for the UNI-VG model. To this end, we adopt a simulation approach. We construct a sample of MBRC by randomly drawing product characteristics and parameters of the underlying price processes from a uniform range of admissible 'realistic' values which are consistent with the calibration in the following sections. The upper and lower boundaries of the admissible ranges are chosen as: $0.1 \leq T \leq 1 ; 0.6 \leq H \leq 0.8 ; 0.05 \leq \nu \leq 0.5 ; 0.05 \leq \omega_{i} \leq 0.4 ;-0.6 \leq \theta_{i} \leq 0 ;$ and $0 \leq c_{i j} \leq 0.8$, where $T$ denotes the time to maturity, $H$ is the downside barrier and $c_{i j}$ are pairwise correlations. We assume that there are 3 underlying stocks with initial stock prices of 1 . The strikes are equal to the initial stock prices. The parameters $T, H$, and $\nu$ are MBRC-specific, while the other parameters are drawn independently for each stock $\left(\omega_{i}, \theta_{i}\right)$ or pair of stocks $\left(c_{i j}\right)$. We apply Monte Carlo Simulation (MCS) with 1000 time steps and 500000 simulated price paths to approximate the theoretical values of the structured products within the VG framework. For comparison, we also compute the theoretical values within the multivariate Black-Scholes 


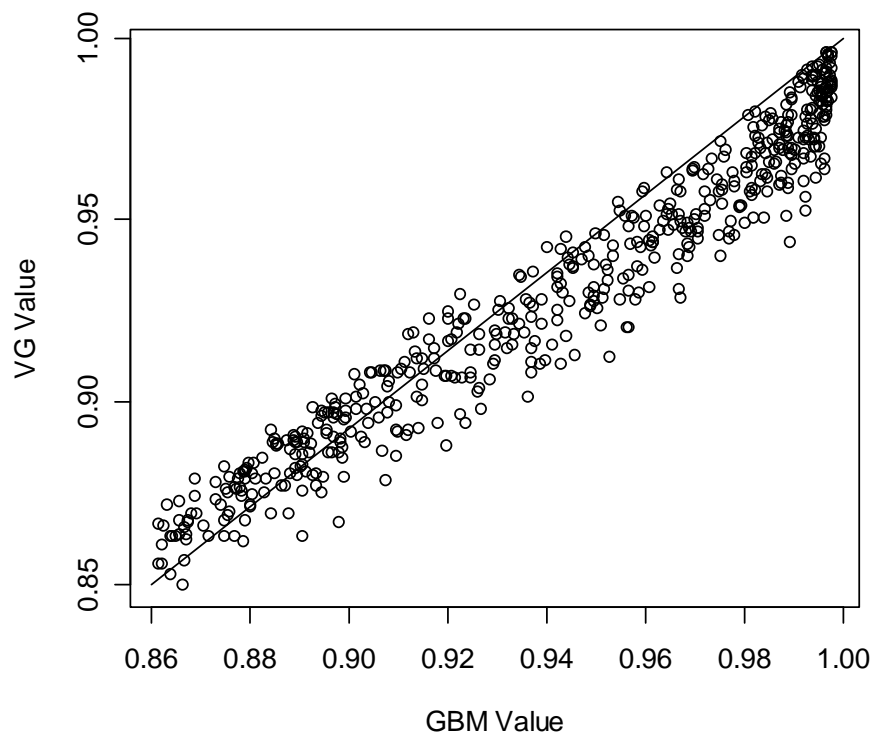

Figure 3: Values of multivariate options obtained with the Geometric Brownian Motion (GBM) and with the Variance Gamma (VG) model

framework using MCS. The volatility parameter of the Geometric Brownian Motion (GBM) is set equal to the Black-Scholes implied volatility of the Variance Gamma value of an at-the-money (ATM) option. Thus, ATM implied volatilities are equal in both models.

In 274 of our 1000 sample cases, the randomly drawn correlations are not attainable within the VG framework, given the other randomly drawn parameters. This means that the respective parameter sets are not consistent with the multivariate VG model. Therefore, we exclude them from the further analysis. Other products have an unrealistically low theoretical value. Typically, the products are sold for a price equal to the nominal value of 1 . This means that the coupon and barrier are adjusted so that a selling price of 1 is achievable. As our simulation does not consider coupon payments, the simulated prices correspond to the present value of repayment at maturity. We restrict this to be at least 0.86 to avoid unrealistically high implicit coupon payments. This gives us a sample of 535 simulated products included in Figure 3. The scatterplot of theoretical values according to the VG and the Normal (Black-Scholes type) models clearly shows that the VG value is in most cases significantly smaller than the GBM value. On average, we obtain a difference of 116 basis points.

Let $\Delta V$ denote the value difference between the two models:

$$
\Delta V=\mathrm{GBM} \text { value }-\mathrm{VG} \text { value. }
$$

As will be explained in the following, we expect $\Delta V$ to increase

- with a higher variance rate of the VG process $\nu(\mathrm{H} 1)$,

- a lower barrier $H(\mathrm{H} 2)$, 
- a shorter time to maturity $T$ (H3),

- lower correlation coefficients $c_{i j}(\mathrm{H} 4)$,

- lower (more strongly negative) skewness skew $_{i}$ (see Eq. 3) implied by the VG processes (H5),

- and lower variances $v a r_{i}$ of the VG processes (see Eq. 2) for given skewness levels (H6).

The variables $s k e w_{i}$ and $v_{a r}$ measure characteristics of the risk-neutral VG process and can therefore be regarded as a representation of the level and slope of the smile pattern in option prices. We do not include risk-neutral kurtosis, because it is strongly (negatively) correlated with skewness in the cross-section of our sample of simulated MBRC. Hypothesis H1 reflects the fact that the VG process is identical to the Geometric Brownian motion in the limit of $\nu \rightarrow 0$. $\mathrm{H} 2$ postulates that the different representations of the tails of the stock return distributions are expected to be more important when the downside barrier is positioned in the tail (low barrier). According to $\mathrm{H} 3$, a longer time to maturity should alleviate the difference, because the smile implicit in the VG process is particularly pronounced for a short time to maturity. Low correlation coefficients render the multi-asset characteristic of the product more important, which intensifies the model differences (H4). Skewness is supposed to be particularly relevant, since excess skewness is neglected in the Normal framework (H5). We expect its influence to be larger for low variance levels (H6). Since variance and skewness have a sample correlation below -0.6 , we define skew in relative terms as the ratio of (risk-neutral VG) skewness and variance. Thus, variables $v a r_{i}$ and $s_{k e w}$ are defined as:

$$
\begin{aligned}
\operatorname{var}_{i} & =\omega_{i}^{2}+\nu \theta_{i}^{2} \\
\operatorname{skew}_{i} & =\left(2 \theta_{i}^{3} \nu^{2}+3 \omega_{i}^{2} \theta \nu\right) / v^{2} r_{i}
\end{aligned}
$$

The pairwise correlations between the explanatory variables in the cross-section of our sample are generally low with one exception: the variance rate $\nu$ has a correlation to all three skew variables of above 0.5. This is not surprising, given the formula for skew. Thus, we discard $\nu$ from the analysis. We estimate the following regression equation:

$$
\begin{aligned}
\Delta V & =\beta_{0}+\beta_{1} H+\beta_{2} T+\beta_{3} c_{12}+\beta_{4} c_{13}+\beta_{5} c_{23} \\
& +\beta_{6} \operatorname{var}_{1}+\beta_{7} \text { var }_{2}+\beta_{8} \text { var }_{3}+\beta_{9} \text { skew }_{1}+\beta_{10} \text { skew }_{2}+\beta_{11} \text { skew }_{3}+\epsilon
\end{aligned}
$$

where $\beta_{0}, \ldots, \beta_{11}$ are regression coefficients and $\epsilon$ is an error term. It is apparent from the results in Table 1 that the hypotheses are confirmed on high significance levels. Only the impact of the pairwise correlations is rather small and in only one case statistically significant. Our regression model explains about $63 \%$ of the variation of the differences between the VG and Normal valuation. 


\begin{tabular}{lrrr}
\hline & Coeff. & t-value & Sig. \\
\hline$H$ & -0.360 & -13.214 & $<0.001$ \\
$T$ & -0.297 & -10.748 & $<0.001$ \\
$c_{12}$ & -0.019 & -0.703 & 0.482 \\
$c_{13}$ & -0.080 & -2.989 & 0.003 \\
$c_{23}$ & 0.020 & 0.725 & 0.469 \\
var $_{1}$ & -0.190 & -5.629 & $<0.001$ \\
var $_{2}$ & -0.151 & -4.364 & $<0.001$ \\
var $_{3}$ & -0.125 & -3.607 & $<0.001$ \\
skew $_{1}$ & -0.370 & -9.986 & $<0.001$ \\
skew $_{2}$ & -0.316 & -8.372 & $<0.001$ \\
skew $_{3}$ & -0.380 & -9.738 & $<0.001$ \\
\hline$R_{\text {adj }}^{2}$ & \multicolumn{3}{|}{} \\
\hline
\end{tabular}

Table 1: Regression results of explaining the difference in option values between the UNI-VG model and Geometric Brownian motion

\section{Empirical analysis}

\subsection{Data}

Our database consists of all MBRCs with three underlying stocks outstanding at the end of April 2007. These are called Triple Barrier Reverse Convertible (TBRC). We collected the product characteristics from their final termsheets. All products are designed such that the issuing price is equal to the nominal value (of 100). We only consider products with stocks as underlying assets, and require Eurex options on these stocks to be available. In addition, we exclude TBRCs with an initial time to maturity of more than 430 calendar days because trading activity is very low in such long-term Eurex options. Starting with 832 stock-based MBRCs, the time-to-maturity requirement reduces this number by 76 , and the Eurex option condition by another 288 . Thus, our final database consists of 468 MBRCs.

The five most important issuers are UBS, Clariden Leu, Julius Bär, Goldman Sachs and Credit Suisse (see Table 2). The "big five" stocks in the Swiss Market Index (Credit Suisse Group, Nestlé, Novartis, Roche Holding, UBS), plus ABB and Zurich Financial Services, are the most frequently used underlying assets in our final database.

Barriers range from $50 \%$ to $85 \%$ of stock prices at issuance (fixing prices). There is a clear preference for round numbers or one percentage point less, for instance $69 \%$ and $70 \%$. Some issuers pay the whole coupon at maturity, while others split it into several parts. To make the payments comparable, we discount coupons to the issuance date. The discounted coupons range from $3.3 \%$ to $17 \%$ of nominal, with an average of $10.4 \%$.

We assume dividend yields and dividend payment dates to be the same as in 2006. Riskfree rates are defined as the 12-month interbank offered rates in the corresponding currency. Credit spreads at the valuation date are assumed to be equal to the premium of credit default 
swaps on senior debt of the issuer. If this information is not available from Thomson Financial Datastream or Bloomberg, we assume a constant credit spread of 25 basis points which appears to be a realistic estimate for the time period under study.

Based on Eurex settlement data, we calculate implied volatilities for all strike prices and maturities available. To account for the American style of Eurex stock options, we apply the binomial model to determine implied volatilities, i.e., we evaluate an option with different volatility input parameters until convergence to the settlement price is achieved. We then apply a two-step interpolation scheme to match the moneyness and maturity dates of MBRCs and Eurex options. In the first step, following Hafner and Wallmeier (2001), we estimate the smile function for each option maturity based on a cubic regression of implied volatilities on moneyness. In the second step, we linearly interpolate between the implied variances of the smile functions surrounding the maturity date of the MBRCs. We use the at-the-money implied volatility as an estimate of the volatility of $\log$ stock returns in the numerical valuation of MBRCs. Since market estimates of correlations are not available, we compute historical correlations of daily log returns over the last year.

\begin{tabular}{llr}
\hline Issuer & Product name & Number of MBRCs \\
\hline UBS & Kick-In GOAL on Worst of & 95 \\
Clariden Leu & Multi Barrier Reverse Convertible & 76 \\
Julius Bär & Triple ICE Unit & 58 \\
Credit Suisse Group & Equity Yield Note & 45 \\
Bank Vontobel & Multi Defender Vonti & 45 \\
Zürcher Kantonalbank & Soft-Runner on Worst of & 37 \\
Goldman Sachs & Multi Barrier Reverse Convertible & 33 \\
Banque Cantonale Vaudoise & LEMAN DEFENSIF WORST OF & 22 \\
Bank Sarasin & SaraSail Rainbow mit Barriere & 20 \\
Citigroup & Barrier Reverse Convertible & 17 \\
Merrill Lynch & Callable Worst of Kick-in Reverse Convertible & 10 \\
Sal. Oppenheim & BARRIER-TRIPLE-REVERSE-CONVERTIBLE & 10 \\
\hline Total & & 468 \\
\hline
\end{tabular}

Table 2: Issuer, product name and number of TBRCs in the final database

\subsection{Smile-consistent Calibration}

We apply the method proposed by Leoni and Schoutens (2008) to calibrate the UNI-VG model of Section 2.1. The approach uses two types of input data: (1) the estimated marginal riskneutral distributions of the underlying stock returns as represented in the strike price profiles of implied volatilities, and (2) the estimated pairwise correlations of stock returns.

In the first step of the procedure, the data of type (1) are used to find the optimal set of parameters $\left(\nu, \omega_{i}, \theta_{i}\right)$ for $i=1,2,3$. Since $\nu$ is the only common parameter, for given $\nu$, the calibration can be carried out separately for each stock. Thus, we can employ the standard 
Carr-Madan optimization method with root mean squared error (RMSE) of implied volatilities as error criterion. We then calculate the average RMSE from the three underlying stocks as a global error measure. The procedure is repeated for various $\nu$ values to find the parameter set with the minimal global error.

In the second step, the correlations of the Brownian motions in the VG process are fixed such that the asset correlations are equal to historical correlations (input data of type (2)). In the VG model, both the correlations of Brownian motions and the joint dependence on $V$ (with stock specific sensitivities $\theta$ ) contribute to the stock return correlations. To formalize this, let the vector of stock return volatilities be defined as $\sigma_{\text {assets }}=\left(\sqrt{\sigma_{1}^{2}}, \ldots, \sqrt{\sigma_{k}^{2}}, \ldots, \sqrt{\sigma_{n}^{2}}\right)^{\prime}$, where $\sigma_{k}^{2}=\omega_{k}^{2}+\nu \theta_{k}^{2}(k=1,2,3)$ are stock return variances. We use these volatilities to transform the correlation matrix $\mathbf{C}_{\text {assets }}$ of stock returns (input data) to the corresponding covariance matrix $\Psi_{\text {assets }}=\sigma_{\text {assets }} \sigma_{\text {assets }}^{\prime} \otimes \mathbf{C}_{\text {assets }}$ where $\otimes$ denotes element-wise multiplication. Then, the correlation matrix of Brownian motions in the multivariate VG model is given by:

$$
\mathbf{C}_{B R}=\left(\Psi_{\text {assets }}-\nu \theta \theta^{\prime}\right) \oslash\left(\omega \omega^{\prime}\right)
$$

with $\omega=\left(\omega_{1}, \ldots, \omega_{n}\right)^{\prime}, \theta=\left(\theta_{1}, \ldots, \theta_{n}\right)^{\prime}$ and $\oslash$ denoting element-wise division.

The resulting parameter set is used to simulate stock price processes and to evaluate MBRC using Monte Carlo Simulation.

To calibrate the $\alpha$-VG model, the marginal parameters $\left(\alpha_{i}, \omega_{i}, \theta_{i}\right)$ are obtained in the first step from replicating the smile in option prices. In the second step, the single common parameter $a$ is fixed such that the average pairwise correlation implied by the $\alpha$-VG model is equal to the average empirical correlation. ${ }^{10}$ Whereas the overall correlation level can be adjusted using parameter $a$, the correlation structure is fixed by the marginal parameters. Thus, the empirically estimated correlations cannot be perfectly replicated in this approach. We only accept calibrations where each pairwise correlation lies within the $90 \%$ confidence interval of the empirical estimate.

The stepwise calibration method which we use for both models is subject to two main weaknesses (see Embrechts et al. (2002)). First, the multivariate distribution is not uniquely determined by the marginal distributions and the pairwise correlations. In particular, our choice of the VG model imposes a structure on the multivariate distribution that is not necessarily compatible with the data even if the marginal distributions and the pairwise correlations are perfectly matched. Second, as already mentioned, for given marginal distributions, the estimated stock return correlations are not always attainable.

In spite of these weaknesses, the stepwise approach is appealing due to the following characteristics. First, it uses option market prices to obtain estimates of the marginal distributions. In many cases, this will provide more accurate results than estimations based on historical stock

$\overline{{ }^{10} \text { As in the UNI-VG case, we take historical }}$ correlations as proxies for risk-neutral ones. 
return data. Second, previous research has shown that the VG model captures important characteristics of stock returns in the univariate case (see Madan et al. (1998)). Therefore, it is a promising candidate for a multivariate extension. Third, the implementation is relatively simple due to the fact that smile replication and correlation fit can be separated. To efficiently implement the calibrations, financial institutions could build up a database of smile characteristics for a wide range of marginal parameters. When such a database is available, the best fitting, smile-consistent set can be found by a grid search among the stored smile profiles. Taken together, one strength of the method is that it has a chance of widespread application if it turns out to be a significant improvement over the multivariate normal model.

The comparison of our two models can be summarized as follows: The $\alpha$-VG model allows a better smile replication in the first step, because the variance rate of the subordinator is stockspecific. In addition, it is compatible with low correlations which can easily be achieved by chosing an $a$-value near zero. In the UNI-VG model, low correlations cannot be achieved if the smile profiles are pronounced and the parameters are similar for the underlying assets. If correlations are high, however, the UNI-VG model is better positioned for two reasons: first, the correlations of the Brownian motions can be used to increase the asset correlations, second, the upper bound of the $a$-parameter in the $\alpha$-VG model is determined by the maximum of the $\alpha_{i}$-parameters, which can be rather restrictive. In addition, the structure of the correlation matrix is fixed by the marginal parameters, whereas the UNI-VG model is more flexible due to the correlated Brownian motions. Both models are parsimonious and easy to implement. The number of parameters is the same if we only consider 3-dimensional processes (parameters $\alpha_{1}, \alpha_{2}, \alpha_{3}$, and $a$ for $\alpha$-VG instead of $\nu$ and three Brownian correlations for UNI-VG).

\subsection{Specification Analysis: Smile-consistency}

\begin{tabular}{clccccc}
\hline & & $\begin{array}{c}-0.25 \leq M \\
<-0.15\end{array}$ & $\begin{array}{c}-0.15 \leq M \\
<-0.05\end{array}$ & $\begin{array}{c}-0.05 \leq M \\
<0.05\end{array}$ & $\begin{array}{c}0.05 \leq M \\
\leq 0.15\end{array}$ & $\begin{array}{c}0.15 \leq M \\
\leq 0.25\end{array}$ \\
\hline \multirow{2}{*}{1} & \multirow{2}{*}{ Empirical smile $(\overline{I V})$} & 0.2580 & 0.2359 & 0.2221 & 0.2137 & 0.2097 \\
& $(0.0483)$ & $(0.0435)$ & $(0.0415)$ & $(0.0402)$ & $(0.0393)$ \\
\hline 2 & MDiff GBM & -0.0359 & -0.0138 & 0 & 0.0084 & 0.0124 \\
3 & MDiff UNI-VG & -0.0076 & -0.0007 & 0 & 0.0042 & 0.0086 \\
4 & MDiff $\alpha$-VG & -0.0051 & 0.0006 & 0 & 0.0039 & 0.0082 \\
\hline 5 & MMaxDiff GBM & 0.0514 & 0.0193 & 0 & 0.0121 & 0.0189 \\
6 & MMaxDiff UNI-VG & 0.0182 & 0.0034 & 0 & 0.0117 & 0.0178 \\
7 & MMaxDiff $\alpha$-VG & 0.0139 & 0.0011 & 0 & 0.0088 & 0.0124 \\
\hline 8 & RMSE GBM & 0.0545 & 0.0205 & 0 & 0.0125 & 0.0193 \\
9 & RMSE UNI-VG & 0.0267 & 0.0138 & 0 & 0.0113 & 0.0141 \\
10 & RMSE $\alpha$-VG & 0.0114 & 0.0066 & 0 & 0.0066 & 0.0092 \\
\hline
\end{tabular}

Table 3: Accuracy of smile replication. 
In this section, we compare the empirical smile profiles with the corresponding profiles implied by our different pricing models. Table 3 shows an aggregated analysis of the smile pattern, where averaging is done across stocks and time-to-maturity. The average implied volatilities $\overline{I V}$ in the first row are defined as:

$$
\overline{I V}_{i}=\frac{1}{N_{i}} \sum_{s \in \mathcal{S}} \sum_{0.25 \leq \tau \leq 1} I V_{s \tau i}
$$

where $I V_{s \tau i}$ is the empirically observed implied volatility of a plain-vanilla option with time-tomaturity $\tau$, underlying stock $s$ and a moneyness within moneyness class $i, i=1, \ldots, 5$. The stock sample $\mathcal{S}$ corresponds to all stocks used as underlying assets in our sample of TBRC, and the time-to-maturity varies between three months and one year. The number of implied volatilities in moneyness class $i$ is $N_{i}$. We use a time-to-maturity adjusted moneyness $M$ defined as:

$$
M=\frac{\ln \left(X e^{-r T} / S\right)}{\sqrt{T}}
$$

where $T$ is the option time-to-maturity, $X$ the exercise price, $S$ the current stock price and $r$ the risk-free rate.

The other statistics in Table 3 are defined as follows: MDiff is the average difference between implied volatilities of a calibrated pricing model and the empirically observed implied volatilities. As before, averaging is done within each moneyness class across stocks and time-to-maturity. MMaxDiff is defined as the average absolute difference between fitted and empirical implied volatilities for each moneyness class, but only for a subsample of stocks. For each TBRC, only the stock with the largest deviation between fitted and empirical smile is considered in computing MMaxDiff. RMSE is the root mean squared error of implied volatilities when all stocks are included.

As can be seen from row 1 of Table 3, the implied volatilities, on average, exhibit the typical moneyness pattern, with a high value for far OTM puts. The smile is significant and economically important, but not as pronounced as in stock index options. The standard deviations of observed implied volatilities in moneyness class $i$ are given in parentheses.

The GBM assumption implies that implied volatilities are flat. Thus, the MDiff values for this model correspond to the difference between the assumed constant volatility (equal to the ATM implied volatility) and the empirical implied volatility in moneyness class $i$. It is evident that this deviation is systematically related to moneyness.

For far OTM puts, the implied volatilities from the VG models are still smaller on average than the empirically observed values, but the difference is strongly reduced compared to the Gaussian model (see rows 2 and 3). For example, while the Gaussian model underestimates the implied volatilities of moneyness class $i=1$ by 3.59 percentage points, the deviation is reduced to 0.76 for UNI-VG and 0.51 percentage points for $\alpha$-VG. 
This ranking of our pricing models is confirmed by the MMaxDiff and RMSE statistics: UNIVG turns out to be an improvement over GBM, but $\alpha$-VG provides an even better fit to the smile patterns, with significantly smaller MMaxDiff and RMSE values compared to UNI-VG. This is not surprising, because $\alpha-\mathrm{VG}$ allows the variance rate to be stock-specific, while it is a common parameter in the UNI-VG model. Our analysis shows that this advantage is relevant and important.

\subsection{Empirical Results}

Given the parameters which result in the best fit to the smile profiles, the UNI-VG and $\alpha$-VG model are compatible with empirical correlation data in only 277 and 218 out of 468 TBRC in our sample. In all other cases, the correlation estimates could not be achieved given the marginal parameters from fitting the observed smile patterns. For an intersection of 179 products, a calibration consistent with the empirical data is found for both models. In these cases, the resulting model values are very similar. The $\alpha$-VG model is able to evaluate 39 additional TBRC, which tend to be products with low correlations of the underlying assets. The 98 cases in which only the UNI-VG model provides a value are typically characterized by higher than average correlations and a less pronounced smile profile.

Thus, there is a tradeoff between the two VG models: one $(\alpha-\mathrm{VG})$ performs better in capturing the smile (see section 4.3), the other (UNI-VG) is more often able to capture the correlation structure. However, it is important to note that the performance of the $\alpha$-VG model in capturing the correlation structure could be improved by accepting a less than perfect smile mapping. In fact, higher correlations could be achieved by reducing the highest $\alpha_{i}$-coefficient in order to increase the adjustment parameter $a$ (see section 2.2$)^{11}$. No similar improvement is possible for UNI-VG. Thus, if the user is content with the precision of smile fitting provided by UNI-VG, the $\alpha$-VG model can be optimized so that it captures higher correlations than in our implementation. Therefore, we have to be cautious in interpreting the number of TBRC which can be evaluated in our framework. The lower number for $\alpha$-VG compared to UNI-VG is a reflection of a higher precision the $\alpha$-VG model achieves at the marginal level.

Descriptive statistics of the estimated parameters for both models are given in Table 4 . The average variance rate is 0.21 versus $0.25,{ }^{12}$ the $\omega$ parameters are clearly positive with mean values of about 0.22 and standard deviations of about 0.05 . The negative theta coefficients indicate the prevailing skew in option implied volatilities. The historical correlations $\left(c_{i j}\right)$ of stock returns are all positive and vary between 0.07 and 0.78 with an average of approximately 0.4 to 0.45 . Overall, these estimates are in line with expectations and previous research (see Leoni and Schoutens (2008)). However, a major limitation is that only 316 out of 468 TBRC

\footnotetext{
${ }^{11}$ See also section 5 in Luciano and Semeraro (2010b) for a generalization which is consistent with higher correlations.

${ }^{12}$ For the $\alpha$-VG model, the average variance rate $\bar{\alpha}=\left(\alpha_{1}+\alpha_{2}+\alpha_{3}\right) / 3$ is given in Table 4 .
} 


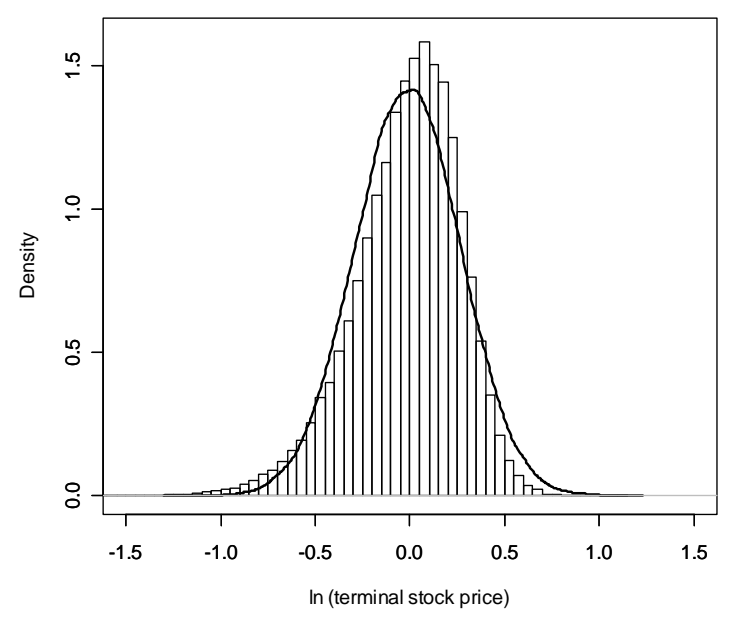

Figure 4: Typical distribution of log stock prices at maturity according to VG and GBM

can be evaluated with at least one of the two models. This clearly shows that there is a need for generalisations which alleviate the correlation problem, such as the models proposed by Luciano and Semeraro (2010b) and Marfè (2009).

Figure 4 illustrates the typical distribution of log stock prices at maturity according to the VG and the GBM model, where the VG parameters are equal to the mean values in Table 4 (model UNI-VG). Clearly, the VG model captures the stylized fact of leptokurtosis of stock returns, and in particular implies a fatter tail on the left.

\begin{tabular}{lrrrr|rrrr}
\hline & \multicolumn{4}{c|}{ UNI-VG $(N=277)$} & \multicolumn{4}{c}{$\alpha$-VG $(N=218)$} \\
\hline & Min & Max & Mean & Std.Dev. & Min & Max & Mean & Std.Dev. \\
\hline$\nu ; \bar{\alpha}$ & 0.15 & 0.35 & 0.2103 & 0.0236 & 0.05 & 0.75 & 0.2463 & 0.0431 \\
$\omega_{1}$ & 0.10 & 0.38 & 0.2313 & 0.0521 & 0.13 & 0.41 & 0.2264 & 0.0554 \\
$\omega_{2}$ & 0.12 & 0.37 & 0.2225 & 0.0535 & 0.13 & 0.37 & 0.2157 & 0.0506 \\
$\omega_{3}$ & 0.14 & 0.45 & 0.2422 & 0.0486 & 0.15 & 0.37 & 0.2387 & 0.0463 \\
$\theta_{1}$ & -0.60 & 0.00 & -0.3152 & 0.1442 & -0.70 & -0.02 & -0.3454 & 0.1327 \\
$\theta_{2}$ & -0.70 & 0.00 & -0.2791 & 0.1694 & -0.60 & 0.00 & -0.3234 & 0.1413 \\
$\theta_{3}$ & -0.70 & 0.00 & -0.3350 & 0.1721 & -0.70 & -0.01 & -0.3697 & 0.1459 \\
$c_{12}$ & 0.12 & 0.78 & 0.4338 & 0.1363 & 0.12 & 0.78 & 0.4121 & 0.1289 \\
$c_{13}$ & 0.07 & 0.77 & 0.4559 & 0.1491 & 0.07 & 0.73 & 0.4275 & 0.1390 \\
$c_{23}$ & 0.14 & 0.77 & 0.4280 & 0.1397 & 0.14 & 0.77 & 0.3979 & 0.1256 \\
$H$ & 0.57 & 0.85 & 0.7476 & 0.0469 & 0.57 & 0.85 & 0.7510 & 0.0467 \\
\hline
\end{tabular}

Table 4: Descriptive statistics of estimated parameters of multivariate Variance Gamma processes

Table 5 shows descriptive statistics for the values of the multivariate barrier put embedded in the MBRCs. To be able to compare the Variance Gamma values with values from the GBM model, we ensure that implied volatilities of single stock ATM options are equal in both models for all three underlying stocks. Since the time to maturity of the MBRC in our sample is one 
year, the put value (VGPut vs. GBMPut) can be interpreted as the present value of the annual coupon surplus that is necessary to compensate investors for selling the embedded put. This compensation varies between $3 \%$ and $23 \%$ with a mean of about $14 \%$ and a standard deviation of about $3 \%$. Thus, the put value is substantial in most cases, allowing banks to offer high coupons. On average, the put value according to the VG model is only slightly higher than the GBM based value. The mean value difference of 37 basis points (UNI-VG) or 80 basis points $(\alpha-\mathrm{VG})$ is statistically significant, but small in economic terms given the typical overpricing of such products of about 3 to $6 \%$. Figure 5 shows the scatterplot of UNI-VG model values (upper left graph) and the histogram of value differences (GBM minus UNI-VG, upper right graph). The corresponding graphs for $\alpha-\mathrm{VG}$ instead of UNI-VG (not shown) look very similar. The illustrations confirm that the option values generated by the GBM and VG are closely related. In all, these results seem to be in contrast to our simulation in Section 3.2 which demonstrated considerable differences between the models. In addition, it seems to be counter-intuitive that the fatter tails of the VG distribution apparently do not translate into higher downside risk.

To explain these empirical results, we decompose the put option value into two components. Part 1 is the put value when only considering cases where the minimal terminal stock price is below the barrier. Part 2 is the put value when only considering cases where the barrier has been broken and the minimal terminal stock price lies between the barrier and the strike price. As can be seen from Table 5, Part 1 is typically far more important than Part 2 (mean value of about $12 \%$ compared to about $2 \%$ ). Interestingly, the evaluation of both parts strongly differs between GBM and VG. The VG model provides a significantly higher Part 1-value than GBM, while the GBM model results in significantly higher Part 2-values compared to VG (see Table 5 and the lower scatterplots in Figure 5). Thus, consistent with our expectation, the risk of extremely low stock prices (below the barrier at maturity) is higher in the VG model. However, the VG process generates significantly fewer cases with less extreme negative returns. These differences between the models are also apparent from the probability distribution of the (risk-neutral) minimal log stock price at maturity. Figure 6 shows simulated distributions based on the mean parameter values of Table 4 (model UNI-VG). In contrast to the GBM-based distribution, the VG-based distribution exhibits negative skewness and higher excess kurtosis, which partly explains the different valuation results for the two option components.

So far, our comparison was based on MBRC with a time to maturity of 1 year. We now reevaluate the options based on the same parameters of the stock return processes, but assuming different times to maturity. The initial stock prices are kept at the same level as before. Table 6 shows the results for the UNI-VG model. All observations are the same when we repeat the analysis for the $\alpha$-VG model and the corresponding subsample of TBRC. The first two data columns of Table 6 show average put values for the 277 MBRC for ficticious times to maturity of 0.05 to 1 year. Clearly, the put values decrease with decreasing time to maturity, but the changes are not the same for the GBM and VG models. The value difference tends to increase with decreasing time to maturity in absolute terms, and even more so in relative 


\begin{tabular}{lrrrr|rrrr}
\hline & \multicolumn{4}{c|}{ UNI-VG $(N=277)$} & \multicolumn{4}{c}{$\alpha$-VG $(N=218)$} \\
\hline MGPut & Min & Max & Mean & Std.Dev. & Min & Max & Mean & Std.Dev. \\
GBMPut & 0.0375 & 0.2283 & 0.1449 & 0.0322 & 0.0366 & 0.2705 & 0.1593 & 0.0443 \\
ValueDifference & 0.0260 & 0.2201 & 0.1412 & 0.0330 & 0.0112 & 0.2478 & 0.1513 & 0.0413 \\
VGPutPart1 & 0.0136 & 0.0293 & 0.0037 & 0.0073 & -0.0214 & 0.0520 & 0.0080 & 0.0135 \\
VGPutPart2 & 0.0276 & 0.2067 & 0.1217 & 0.0315 & 0.0269 & 0.2465 & 0.1363 & 0.0442 \\
GBMPutPart1 & 0.0162 & 0.0370 & 0.0233 & 0.0045 & 0.0096 & 0.0482 & 0.0230 & 0.0041 \\
GBMPutPart2 & 0.0098 & 0.0422 & 0.1135 & 0.0327 & 0.0055 & 0.2268 & 0.1235 & 0.0413 \\
\hline
\end{tabular}

Table 5: Decomposition of option values according to the two versions of the VG model and the Geometric Brownian Motion (GBM) model
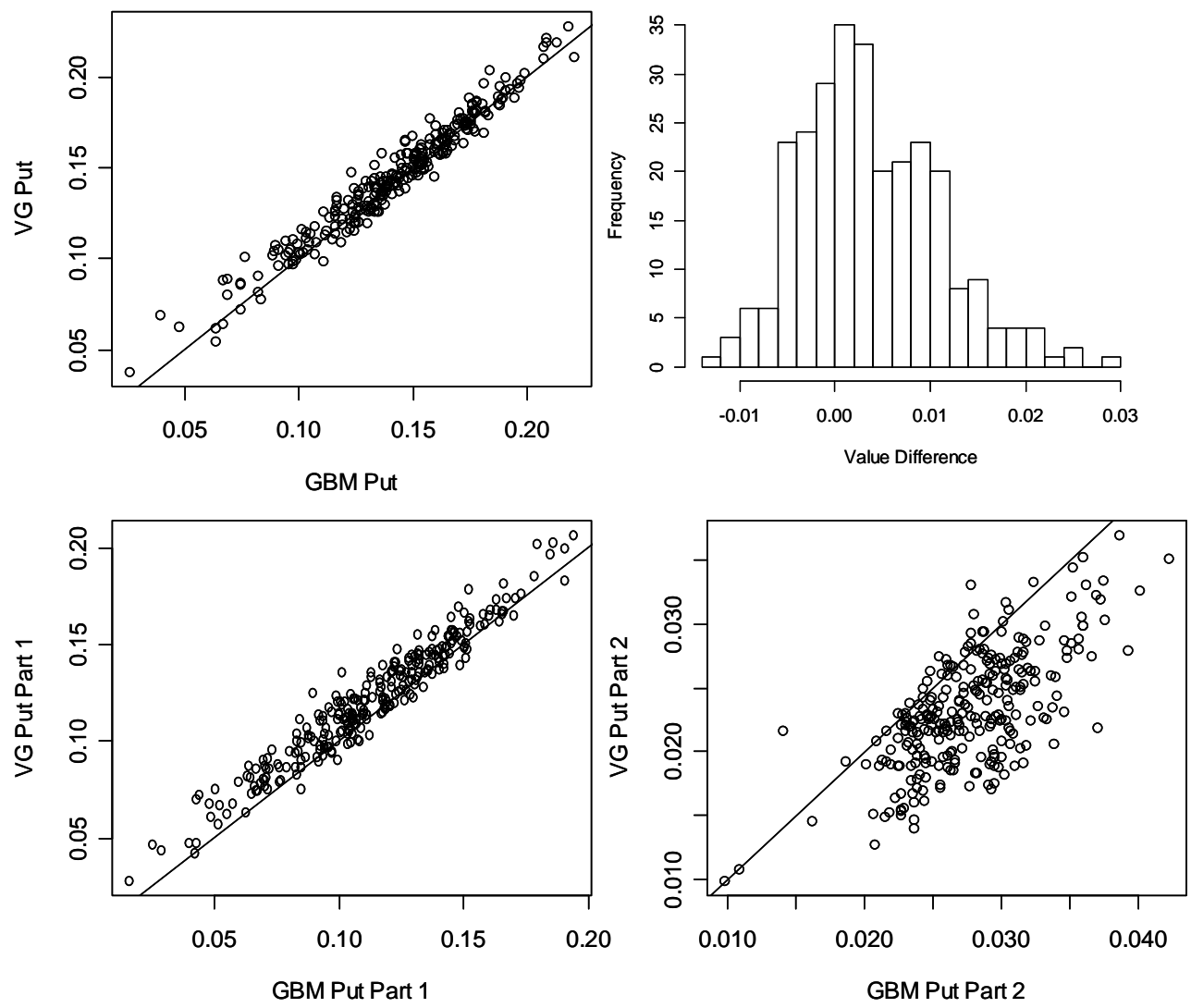

Figure 5: Empirical comparison of put option values obtained with the UNI-VG model and Geometric Brownian Motion (GBM) 

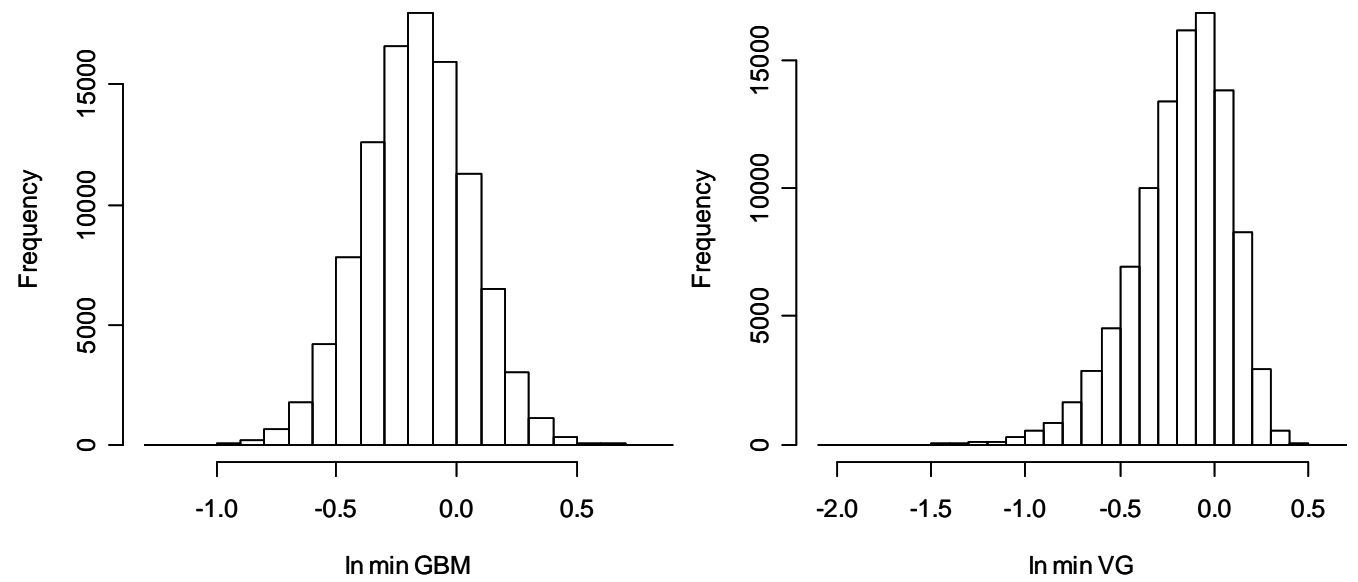

Figure 6: Simulated probability distribution of the (risk-neutral) minimal log stock price at maturity for a process with mean parameter values; left histogram: UNI-Variance Gamma model; right histogram: Geometric Brownian Motion

terms as a percentage of the GBM value (see Table 6 ). For example, for $T=1$, the put value obtained from the VG model is $2.60 \%$ higher than the corresponding GBM value, while the surplus increases to $16.59 \%$ for $T=0.5$ and to almost $100 \%$ for $T=0.25$. These differences are statistically significant and economically important. The strong influence of the time to maturity also explains why the differences between the models were more pronounced in our simulation than in the empirical study. The reason is that the simulation included options with a remaining lifetime between 0.1 and 1 year, while the MBRC had a time to maturity of 1 year.

\section{Conclusion}

Several types of options involving more than one asset are popular in today's financial markets, including rainbow and basket options. In the Swiss market for structured products, one of the most successful instruments is a reverse convertible on multiple underlying stocks (MBRC). It can be regarded as a combination of buying a straight bond and selling a multi-asset put option. Due to the "worst of"-characteristic of the put, the major risk for the holder of an MBRC is a significant downside movement of one of the underlying assets. In valuing the product, it is important to take heavy tails and other stylized facts of asset returns into account. However, modelling financial risk in a multi-asset framework is still a challenge. This explains why the literature on (over-)pricing of structured products typically applies the Black-Scholes framework of log-normal distributions.

A promising candidate for an alternative modelling and valuation framework is the Variance Gamma (VG) model of Madan et al. (1998). In the univariate case, it is well known to be more in line with observed asset returns and option market prices than the Black-Scholes model. But not much is known about the empirical performance of the multivariate extensions developed in 


\begin{tabular}{|c|c|c|c|c|c|c|c|c|}
\hline \multirow[b]{2}{*}{ TTM } & \multirow[b]{2}{*}{ VG Put } & \multirow[b]{2}{*}{ GBM Put } & \multirow{2}{*}{$\begin{array}{r}\text { Value } \\
\text { difference } \\
\text { (absolute) }\end{array}$} & \multirow{2}{*}{$\begin{array}{r}\text { Value } \\
\text { difference } \\
\text { (relative to } \\
\text { GBM Put) }\end{array}$} & \multicolumn{4}{|c|}{ Quantiles of distribution of relative value difference } \\
\hline & & & & & Percentile05 & Percentile25 & Percentile75 & Percentile95 \\
\hline$\overline{T 05}$ & 0.0070 & 0.0002 & 0.0068 & 36.8677 & 4.6958 & 22.0182 & 47.4988 & 84.6485 \\
\hline T10 & 0.0144 & 0.0017 & 0.0127 & 7.3700 & 2.0533 & 4.9063 & 9.8475 & 13.0205 \\
\hline T15 & 0.0236 & 0.0059 & 0.0176 & 2.9742 & 1.0128 & 2.2741 & 3.7664 & 4.5982 \\
\hline T20 & 0.0320 & 0.0135 & 0.0185 & 1.3690 & 0.6952 & 1.1605 & 1.6395 & 1.9882 \\
\hline T25 & 0.0411 & 0.0214 & 0.0196 & 0.9147 & 0.4961 & 0.7505 & 1.0692 & 1.3514 \\
\hline T30 & 0.0511 & 0.0305 & 0.0206 & 0.6759 & 0.3605 & 0.5116 & 0.8017 & 1.0517 \\
\hline T35 & 0.0605 & 0.0378 & 0.0226 & 0.5990 & 0.3417 & 0.4649 & 0.7093 & 0.9081 \\
\hline T40 & 0.0674 & 0.0480 & 0.0194 & 0.4033 & 0.1404 & 0.2967 & 0.5055 & 0.6444 \\
\hline T45 & 0.0719 & 0.0562 & 0.0157 & 0.2799 & 0.0478 & 0.1750 & 0.3689 & 0.5294 \\
\hline T50 & 0.0792 & 0.0680 & 0.0113 & 0.1659 & -0.0289 & 0.0846 & 0.2472 & 0.3614 \\
\hline T55 & 0.0848 & 0.0785 & 0.0063 & 0.0804 & -0.0670 & 0.0118 & 0.1464 & 0.2523 \\
\hline T60 & 0.0921 & 0.0870 & 0.0051 & 0.0580 & -0.0619 & -0.0030 & 0.1115 & 0.2146 \\
\hline T65 & 0.0999 & 0.0976 & 0.0023 & 0.0236 & -0.0892 & -0.0383 & 0.0678 & 0.1770 \\
\hline T70 & 0.1079 & 0.1037 & 0.0043 & 0.0413 & -0.1183 & -0.0178 & 0.0980 & 0.1974 \\
\hline T75 & 0.1157 & 0.1079 & 0.0078 & 0.0722 & -0.0858 & 0.0185 & 0.1326 & 0.2147 \\
\hline T80 & 0.1231 & 0.1139 & 0.0092 & 0.0809 & -0.0820 & 0.0366 & 0.1401 & 0.2398 \\
\hline T85 & 0.1294 & 0.1220 & 0.0074 & 0.0609 & -0.1096 & 0.0102 & 0.1156 & 0.2167 \\
\hline T90 & 0.1349 & 0.1285 & 0.0063 & 0.0494 & -0.0661 & 0.0088 & 0.0893 & 0.1868 \\
\hline T95 & 0.1409 & 0.1352 & 0.0057 & 0.0420 & -0.0334 & 0.0030 & 0.0725 & 0.1481 \\
\hline T Year & 0.1449 & 0.1412 & 0.0037 & 0.0260 & -0.0481 & -0.0088 & 0.0609 & 0.1261 \\
\hline
\end{tabular}

Table 6: Average put values for $277 \mathrm{MBRC}$ with times to maturity of 0.05 to 1 year. VG refers to the UNI-VG model.

recent years. This is the focus of our study. We test two relatively simple, parsimonious models: one proposed by Leoni and Schoutens (2008) with a univariate subordinator and correlated Brownian motions (UNI-VG) and the other proposed by Semeraro (2008) with a multivariate subordinator and uncorrelated Brownian motions $(\alpha-\mathrm{VG})$. We apply these models to a comprehensive sample of MBRC in Switzerland. Our first objective is to evaluate strengths and weaknesses of the models in a large-scale empirical application. The second objective is to contribute to the literature on the pricing of structured financial products by going beyond the standard normal model in considering some important stylized facts of asset returns.

We first perform a simulation study to analyze the differences between the valuation models. For typical parameters, the stock return distributions obtained with the VG processes turn out to be significantly different from those obtained with geometric Brownian motion. The consequences for option values are important. We find that the most important determinants of the value differences are the time to maturity and the degree of skewness in the smile patterns.

In the empirical study, the calibration of the models includes two steps. The first is to find the parameters which best reflect the smiles in option prices. Here, the $\alpha$-VG model offers more flexibility than UNI-VG, because the variance rate can be separately chosen for each underlying stock while it is forced to be equal for all underlying stocks in the UNI-VG model. As a result, $\alpha-\mathrm{VG}$ performs significantly better in capturing the smile patterns. In the second step, the remaining parameters are used to match the model implied asset correlations with 
the empirical correlation estimates. In the $\alpha$-VG model, one adjustment factor is available to shift all correlations upwards or downwards. While low correlations can easily be achieved in this way, the main problem is to capture high correlations. This problem could be alleviated by giving away some precision in the marginal fit. As we do not run such an optimization, our implementation of $\alpha$-VG captures the correlation structure in only 218 out of 468 TBRC. By reducing the precision of smile fitting to the level provided by UNI-VG, this number could clearly be increased. Using UNI-VG, correspondence between empirical and model correlations is achieved in 277 cases.

The results for those cases where the implementation was successful can be summarized as follows. At issuance, the fair values of the MBRC are significantly higher according to the VG model than the normal model. Economic significance, however, is rather small, given a mean difference of 37 or 80 basis points. We show that the results are driven by two opposite effects: on the one hand, the VG model implies a higher probability of strongly negative returns with a final stock price below the barrier; on the other hand, there is a significantly smaller probability of less extreme negative returns. A general statement on the net effect on option valuation is not possible - the model difference will clearly depend on the option characteristics. In the case of MBRC, the time to maturity plays an important role. Near maturity, the normal model sharply underestimates the MBRC put option value.

In all, these results show that non-normality of returns cannot be ignored when valuing multiasset options and should be considered in studies on the overpricing of exotic structured financial products. The multivariate VG model is able to capture important stylized facts, but we find that the VG models considered in this study are rather limited in their potential to match empirically observed correlations. There is obviously a need for more flexible approaches such as those proposed by Marfè (2009) and Luciano and Semeraro (2010b). 


\section{References}

Ané, T. and Geman, H. (1996). Stochastic subordination, RISK 9(9): 145-149.

Ané, T. and Geman, H. (2000). Order flow, transaction clock, and normality of asset returns, Journal of Finance 55: 2259-2284.

Applebaum, D. (2004). Lévy processes: From probability to finance and quantum groups, Notices of the American Mathematical Society 51(11): 1336-1347.

Ballotta, L. (2008). A Note on Multivariate Asset Models Using Lévy Processes, Working Paper Cass Business School.

Barndorff-Nielsen, O. (1997). Normal inverse gaussian distributions and stochastic volatility modelling, Scandinavian Journal of Statistics 24: 1-13.

Barndorff-Nielsen, O. (1998). Processes of normal inverse gaussian type, Finance and Stochastics 2: $41-68$.

Barndorff-Nielsen, O. and Shephard, N. (2001). Modelling by Lévy processes for financial econometrics, in O. Barndorff-Nielsen, T. Mikosch and S. Resnick (eds), Lévy Processes: Theory and Applications, Boston: Birkhäuser, pp. 283-318.

Bibby, B. and Sørensen, M. (2003). Hyperbolic Processes in Finance, Handbook of Heavy Tailed Distributions in Finance, Ed. by S. Rachev, Elsevier, chapter 6, pp. 211-248.

Boyle, P., Evnine, J. and Gibbs, S. (1989). Numerical evaluation of multivariate contingent claims, Review of Financial Studies 2(2): 241-250.

Carr, P., Geman, H., Madan, D. and Yor, M. (2002). The finite structure of asset returns: An empirical investigation, Journal of Business 75: 305-332.

Carr, P., Hogan, A. and Stein, H. (2007). Time for a Change: The Variance Gamma Model and Option Pricing, Working Paper Quantitative Finance Bloomberg LP.

Carr, P. and Madan, D. (1999). Option valuation using the fast Fourier transform, Journal of Computational Finance 2(4): 61-73.

Carr, P. and Wu, L. (2004). Time-changed Lévy processes and option pricing, Journal of Financial Economics 71: 113-141.

Chen, R.-R., Chung, S.-L. and Yang, T. (2002). Option pricing in a multi-asset, complete market economy, Journal of Financial and Quantitative Analysis 37(4): 649-666.

Cont, R. and Tankov, P. (2004). Financial Modelling With Jump Processes, Chapman Hall/CRC.

Eberlein, E. and Keller, U. (1995). Hyperbolic distributions in finance, Bernoulli 1(3): 281-299.

Eberlein, E., Keller, U. and Prause, K. (1998). New insights into smile, mispricing and value at risk: The hyperbolic model, Journal of Business 71: 371-405. 
Embrechts, P., McNeil, A. and Straumann, D. (2002). Correlation and dependence in risk management: Properties and pitfalls, in M. Dempster (ed.), Risk Management: Value at Risk and Beyond, Cambridge University Press, pp. 176-223.

Fiorani, F. (2004). Option Pricing under the Variance Gamma Process, Ph.D. Thesis Università degli Studi di Trieste.

Fiorani, F., Luciano, E. and Semeraro, P. (2010). Single and joint default in a structural model with purely discontinuous asset prices, Quantitative Finance 10(3): 249-263.

Geman, H. (2002). Pure jump Lévy processes for asset price modelling, Journal of Banking and Finance 26: 1297-1316.

Hafner, R. and Wallmeier, M. (2001). The dynamics of DAX implied volatilities, International Quarterly Journal of Finance 1: 1-27.

Henderson, B. and Pearson, N. (2009). The Dark Side of Financial Innovation, Working Paper George Washington University.

Hens, T. and Rieger, M. (2008). The Dark Side of the Moon: Structured Products from the Customer's Perspective, Working Paper University of Zurich.

Hernandez, R., Lee, W. and Liu, P. (2007). An Economic Analysis of Reverse Exchangeable Securities - An Option-Pricing Approach, Working Paper University of Arkansas.

Lam, K., Chang, E. and Lee, M. (2002). An empirical test of the variance gamma option pricing model, Pacific-Basin Finance Journal 10(3): 267-285.

Leoni, P. and Schoutens, W. (2008). Multivariate smiling, WILMOTT magazine 8(March): 8291.

Lindauer, T. and Seiz, R. (2008). Pricing (Multi-) Barrier Reverse Convertibles, Working Paper University of St. Gallen.

Luciano, E. and Schoutens, W. (2006). A multivariate jump-driven financial asset model, Quantitative Finance 6: 385-402.

Luciano, E. and Semeraro, P. (2010a). A generalized normal mean-variance mixture for return processes in finance, International Journal of Theoretical and Applied Finance 13(3): 415440.

Luciano, E. and Semeraro, P. (2010b). Multivariate time changes for Lévy asset model: Characterization and calibration, Journal of Computational and Applied Mathematics 233: 19371953.

Madan, D., Carr, P. and Chang, E. (1998). The variance gamma process and option pricing, European Finance Review 2: 79-105.

Madan, D. and Milne, F. (1991). Option pricing with VG martingale components, Mathematical Finance 1: 39-45. 
Madan, D. and Seneta, E. (1990). The variance gamma (V.G.) model for share market returns, Journal of Business 63(4): 511-524.

Marfè, R. (2009). A Multivariate VG Model for Asset Returns with Multifactorial Dependence Structure, Working Paper, University of Lausanne and Swiss Finance Institute, submitted.

Monroe, I. (1978). Processes that can be embedded in Brownian motion, Annals of Applied Probability 6(1): 42-56.

Raible, S. (2000). Lévy Processes in Finance: Theory, Numerics, and Empirical Facts, Dissertation Universität Freiburg.

Rathgeber, A. (2007). Optionsbewertung unter Lévy-Prozessen, Kredit und Kapital 42(3): 451484.

Rydberg, T. (1999). Generalized hyperbolic diffusion processes with applications in finance, Mathematical Finance 9: 183-201.

Schoutens, W. (2003). Lévy Processes in Finance, Wiley.

Semeraro, P. (2008). A multivariate variance gamma model for financial application, Journal of Theoretical and Applied Finance 11(1): 1-18.

Stoimenov and Wilkens (2005). Are structured products "fairly" priced? an analysis of the German market for equity-linked instruments, Journal of Banking and Finance 29(12): 29712993.

Szymanowska, M., Horst, J. and Veld, C. (2009). Reverse convertible bonds analyzed, Journal of Futures Markets 29(10): 895-919.

Wallmeier, M. and Diethelm, M. (2009). Market pricing of exotic structured products: The case of multi-asset barrier reverse convertibles in Switzerland, Journal of Derivatives 17(2): 5972 . 